\title{
Personalized prescription of tyrosine kinase inhibitors in unresectable metastatic cholangiocarcinoma
}

\author{
Elena V. Poddubskaya1,2, Madina P. Baranova², Daria O. Allina³, Philipp Y. Smirnov, Eugene A. Albert', \\ Alexey P. Kirilchev ${ }^{4}$, Alexey A. Aleshin ${ }^{5}$, Marina I. Sekacheva ${ }^{1}$ and Maria V. Suntsova ${ }^{6 *}$
}

\begin{abstract}
Background: Cholangiocarcinoma is an aggressive tumor with poor prognosis. Most of the cases are not available for surgery at the stage of the diagnosis and the best clinical practice chemotherapy results in about 12-month median survival. Several tyrosine kinase inhibitors (TKIs) are currently under investigation as an alternative treatment option for cholangiocarcinoma. Thus, the report of personalized selection of effective inhibitor and case outcome are of clinical interest.

Case presentation: Here we report a case of aggressive metastatic cholangiocarcinoma (MCC) in 72-year-old man, sequentially treated with two targeted chemotherapies. Initially disease quickly progressed during best clinical practice care (gemcitabine in combination with cisplatin or capecitabine), which was accompanied by significant decrease of life quality. Monotherapy with TKI sorafenib was prescribed to the patient, which resulted in stabilization of tumor growth and elimination of pain. The choice of the inhibitor was made based on high-throughput screening of gene expression in the patient's tumor biopsy, utilized by Oncobox platform to build a personalized rating of potentially effective target therapies. However, time to progression after start of sorafenib administration did not exceed 6 months and the regimen was changed to monotherapy with Pazopanib, another TKI predicted to be effective for this patient according to the same molecular test. It resulted in disease progression according to RECIST with simultaneous elimination of sorafenib side effects such as rash and hand-foot syndrome. After 2 years from the diagnosis of MCC the patient was alive and physically active, which is substantially longer than median survival for standard therapy.
\end{abstract}

Conclusion: This case evidences that sequential personalized prescription of different TKIs may show promising efficacy in terms of survival and quality of life in MCC.

\section{Background}

Cholangiocarcinoma (CCA) is a bile duct cancer that is mainly characterized by its late diagnosis and fatal outcome [1]. CCA accounts about 3\% of all gastrointestinal tumors and is second most common liver tumor after hepatocellular carcinoma [2]. Overall 5-year survival rate is lower than $10 \%$ [3], while overall 1-year survival of patients at stage 4 is only 5\% [4]. Treatment options for

\footnotetext{
*Correspondence: suntsova86@mail.ru

${ }^{6}$ D. Rogachev Federal Research Center of Pediatric Hematology,

Oncology and Immunology, Moscow 117198, Russia

Full list of author information is available at the end of the article
}

CCA include surgery and chemotherapy, but only about $30 \%$ of patients are available for surgery [5].

Standard care chemotherapy treatment for CCA is Gemcitabine, which is administered alone or in combination with cytotoxic agents such as Cisplatin. The response rate ranges from 8 to $60 \%$, depending on the cohort of patients [6]. Nevertheless, these patients have a poor prognosis with a median survival of 6-12 months [7]. Thus, there is a need for improvement of general CCA treatment options.

Currently there are several ongoing clinical trials utilizing different approaches for target therapy in CCA. Chimeric antigen receptor-modified $\mathrm{T}$ (CART) cell-based 
therapy was recently attempted in CCA [8]. Authors reported 8.5- and 4.5-month partial response to CARTEGFR and CART-CD133 therapy respectively. Nevertheless, several associated side effects were discovered, from which epidermal damage was the most prominent. Indeed, so far CART showed limited efficiency in solid tumor and further studies are still required for successful clinical applications [9].

Majority of clinical trials investigate the efficacy of small molecule inhibitors acting at different levels of EGFR signaling pathway, which is known for being upregulated in CCA (summarized in the recent review [10]). Several case reports [11-13] on TKI usage in CCA demonstrated potential benefits from such treatment. However, results of clinical trials so far are controversial. Luo et al. in non-controlled and single arm study showed that Sorafenib in combination with best clinical practice had modest effect for the patients with advanced CCA [14]. At the same time, Sorafenib monotherapy had been shown to be beneficial in a cohort of 15 patients [15], but larger studies demonstrated that Sorafenib as a single agent had rather low activity in cholangiocarcinoma [16, 17]. In turn, Pazopanib in combination with another TKI drug Trametinib showed a trend to increase 4-month progression-free survival as compared with the prespecified null hypothesized 4-month PFS of $25 \%$. However, this trend did not reach statistical significance [18]. Previously reported treatment outcomes described above are summarized in Table 1. Differential efficiency of TKIs between studies and individual patients within a study could be explained by the range of factors from genetic heterogeneity [19] to patient ethnicity [20]. This illustrates that despite the potential benefits for a distinct cohort of CCA patients there are difficulties associated with the correct TKI prescriptions.

In this report we describe a case of advanced metastatic CCA subsequently treated with TKI agents Sorafenib and Pazopanib, which resulted in 2-year survival period after initial diagnosis. Inhibitors were selected based on high throughput gene expression and molecular pathway activation analysis of the patient's tumor biopsy, thus providing a personalized approach.

\section{Case}

The patient is a 72-year-old man with histologically confirmed moderately differentiated intrahepatic cholangiocarcinoma (Fig. 1). He was diagnosed in October 2015 with the following symptoms: moderate weight loss, pain in the right hypochondrium, loss of appetite and asthenia, with a Karnofsky scale index of $70 \%$. MRI image at the time of diagnosis is shown on Fig. 2a. The tumor was not surgically removed because of advanced stage, multiple intrahepatic nodules and lung metastases.
Four courses of chemotherapy ( 2 courses Gemcitabine in combination with Capecitabine and subsequent 2 courses Gemcitabine in combination with Cisplatin) were administered till May 2016. The treatment was poorly effective, and the tumor increased in size according to MRI (Fig. 2b); additional metastatic nodules appeared in the left and the right lobes with the spread to the bile duct, holedoch and into the gallbladder. Serum gamma glutamyltranspeptidase (GGT) level, which is associated with poor prognosis and tumor aggressiveness [21, 22], was significantly increased, when compared to pre-treatment levels (Fig. 3). Karnofsky scale index decreased to $60 \%$. As the patient did not respond to the best clinical practice treatment, we decided to switch the medication and considered TKI inhibitors as further treatment option. Taking into account available data on differential response of CCA patients to TKIs we performed advanced molecular analysis of the tumor to support our choice and identify the most effective drug.

We profiled gene expression in formalin-fixed, paraffin-embedded (FFPE) patient's tumor biopsy sample, obtained at the time of the first CCA diagnosis. Briefly total RNA was extracted using Ambion's RecoverAll ${ }^{\mathrm{TM}}$ Total Nucleic Acid Isolation. Complete Whole Transcriptome Amplification WTA2 Kit (Sigma) was used for reverse transcription and library amplification. Hybridization was performed according to CustomArray ElectraSense ${ }^{\mathrm{TM}}$ Hybridization and Detection protocol. Hybridization efficiency was detected electrochemically using CustomArray ElectraSense ${ }^{\mathrm{TM}}$ Detection Kit and ElectraSense ${ }^{\mathrm{TM}} 4 \mathrm{X} 2 \mathrm{~K} / 12 \mathrm{~K}$ Reader.

We next used bioinformatical software Oncobox to analyze gene expression data and to identify molecular pathways differentially regulated in the patient's tumor sample [23]. Based on the abundance of gene transcripts for the molecular targets of anticancer drugs, Oncobox also makes it possible to generate a rating of target drugs potentially effective for the individual patients $[24,25]$. Particularly, this analysis revealed that the ERK and Ras molecular signaling pathways were highly activated in the CCA patient's tumor biopsy (Fig. 4), the predicted rating of the most effective target drugs is shown in Table 2. Regorafenib, a multi-tyrosine kinase inhibitor was on the top position of the rating. However, there were no published studies of Regorafenib efficacy and tolerability in CCA. At the same time, several case reports demonstrated efficiency of TKI target drug Sorafenib for CCA treatment [11-13]. We, therefore, decided to use Sorafenib as the next line therapy and it was prescribed to the patient (800 $\mathrm{mg}$ daily) in May 2016. Treatment with Sorafenib coincided with the decrease of serum GGT level. MRI analysis in October 2016 revealed moderate tumor growth, corresponding to 


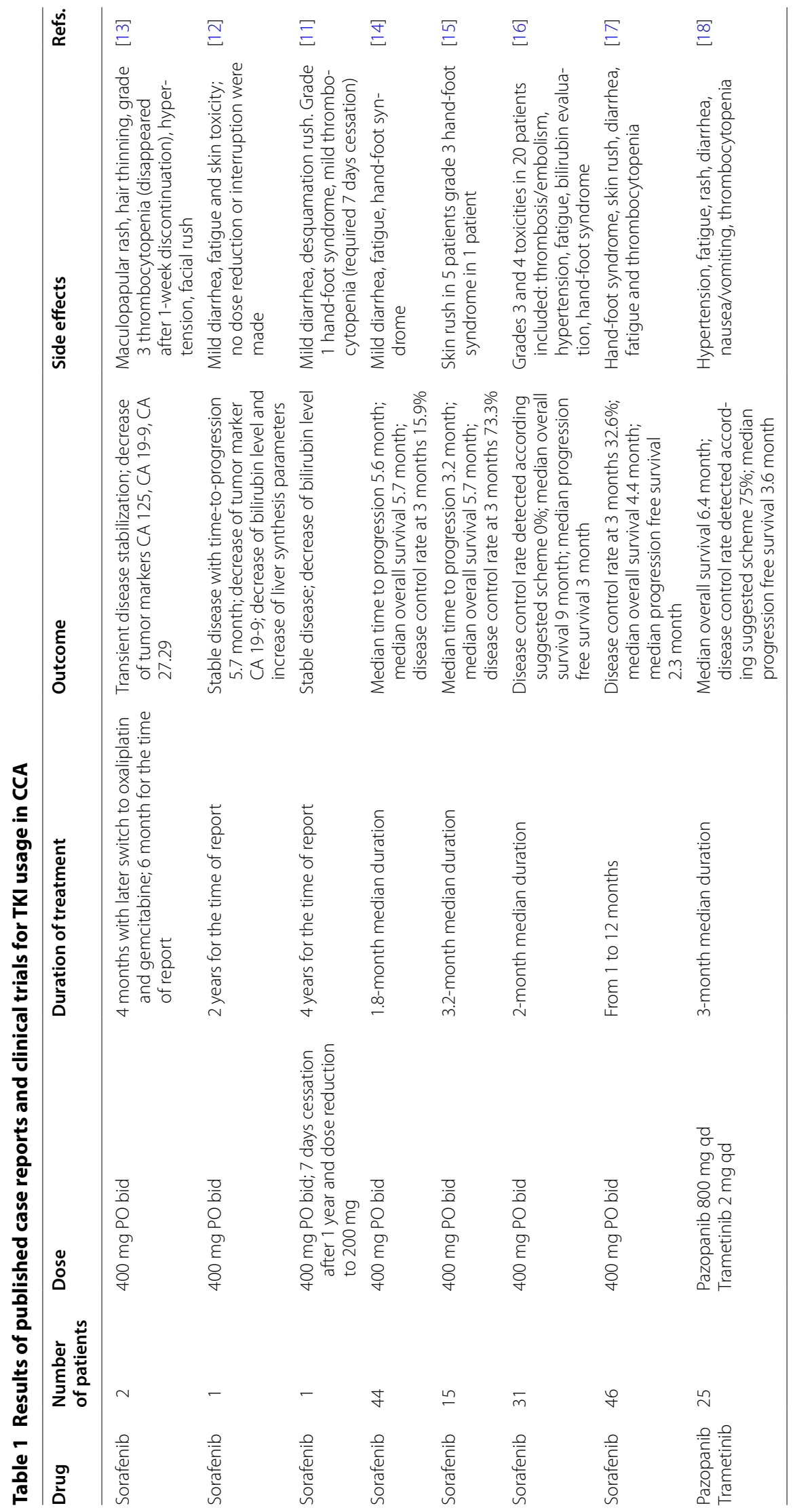




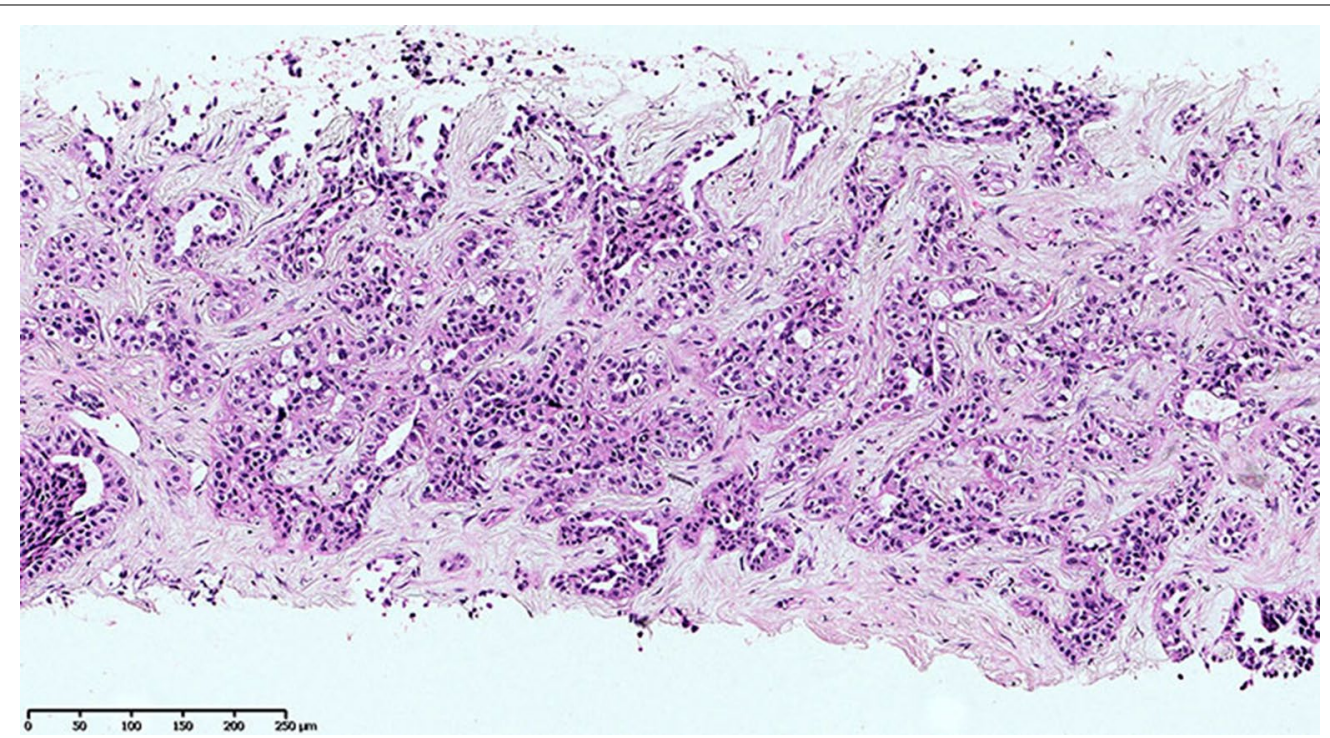

Fig. 1 Hematoxylin and eosin (H\&E) staining shows moderately differentiated intrahepatic cholangiocarcinoma (magnification $\sim \times 200$ )

disease stabilization (Fig. 2c). However, additional nodules occurred slightly below the xiphoid process in the diaphragm area. Therefore, disease progressed according to RECIST criteria. And, importantly, after Sorafenib treatment, the patient did not complain of pain in the right hypochondrium. Before Sorafenib treatment the patient received Tramadol (100 $\mathrm{mg}$ im once a day) and Fentanyl $(75 \mu \mathrm{g} / \mathrm{h}$, Duragesic transdermal tape). After 1 month of treatment with Sorafenib the pain medication was switched to Ketoral (30 mg im twice a day). Considering all the above-mentioned facts it was decided to continue Sorafenib treatment. MRI performed in January 2017 revealed progression of tumor growth and additional nodule in the left lung (Fig. 2d). In addition, the following side effects occurred: redness, swelling, pain on the palms of the hands and soles of the feet. GGT level increased up to 319 U/L in December 2016.

The treatment regimen was next changed to Pazopanib, another TKI drug recommended based on the Oncobox rating. Sunitinib was not chosen because we attempted to eliminate the hand-foot syndrome, which occurred during Sorafenib administration. In the previous studies, Sunitinib treatment of CCA patients induced hand-foot syndrome in $43 \%$ of patients [26]. On the other hand, recent clinical trial of Pazopanib in combination with Trametinib in CCA did not report hand-foot syndrome as a side effect [27]. Pazopanib administration $(800 \mathrm{mg}$ daily) started since January 2017. The control MRI in July 2017 revealed progression in the lung nodes and $20 \%$ increase in sum of diameters of target lesions, which is a borderline between stabilization and progression according to RECIST (Fig. 2e). However, the change of treatment regimen resulted in elimination of Sorafenib side effects and general improvement of life quality. In addition, start of Pazopanib treatment coincided with a start of a trend towards decrease of serum GGT level (Fig. 3). As for October 2017 (2 years after initial diagnosis), the patient was alive and physically active, with Karnofsky scale $80 \%$. Our patient passed away due to the liver failure in November 2017.

\section{Discussion}

Our case report describes sequential use of TKI inhibitors Sorafenib and Pazopanib, which were selected based on personalized approach, for treating advanced CCA in patient who did not respond to standard therapy. Selected treatment improved patient life quality and survival period even though did not result in a response according to RECIST classification.

Available data on TKI usage in CCA patients underline that despite the potential benefit of such treatment not all patients respond equally. Therefore, it remains a clinical challenge to promptly identify potential responders. Selection of CCA patients, who may benefit from TKI treatment may be based on the molecular characteristics of tumor specimens.

Therefore, to support the usage of TKI in the current clinical case and select the most effective inhibitor we performed a molecular profile of patient tumor biopsy. Total RNA was extracted from FFPE tissue sample, obtained at the stage of the diagnosis, and gene 

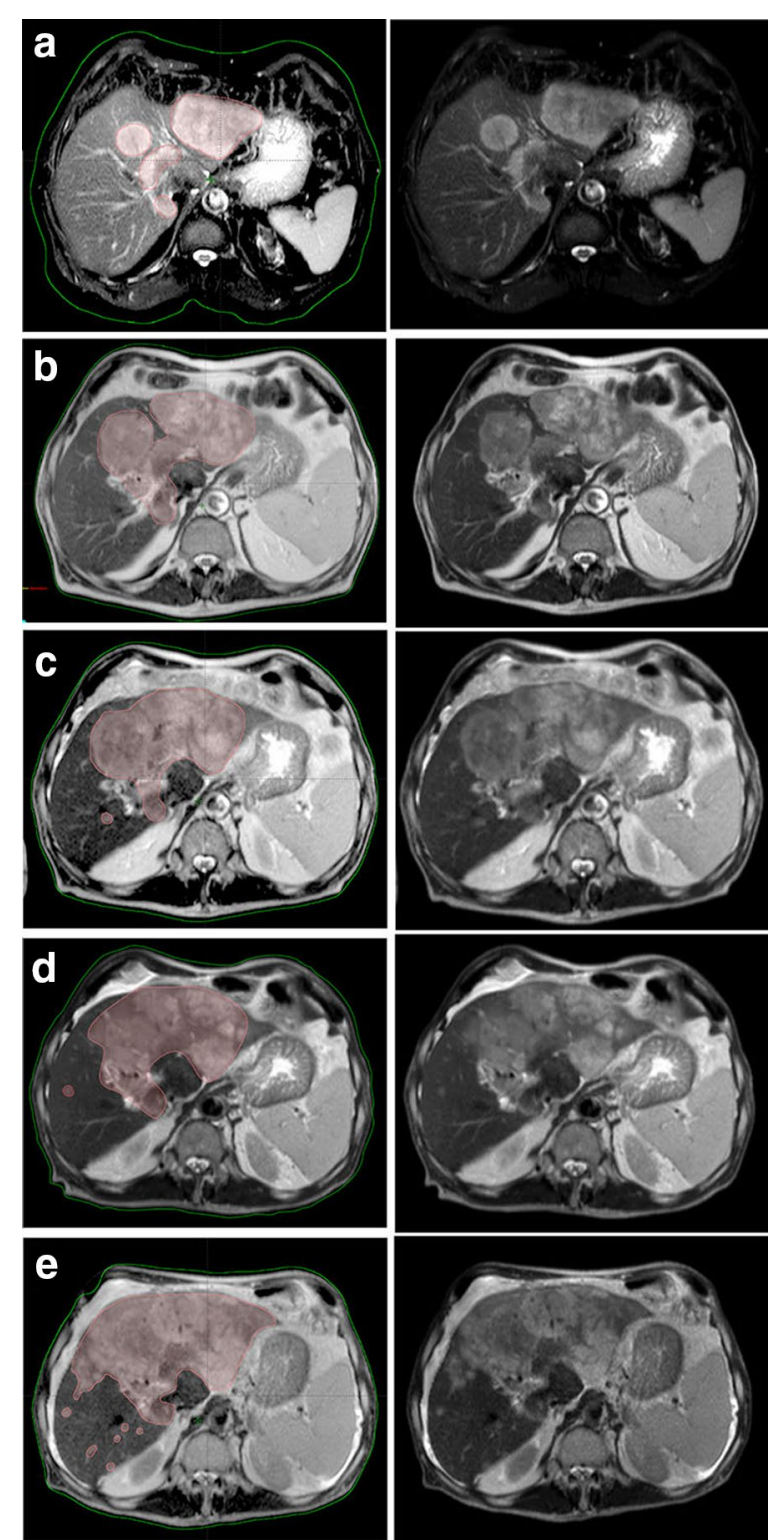

Fig. $2 \mathrm{MRI}$ tumor evaluation during treatment. Tumor area is highlighted on the left panel. Raw images are provided on the right panel. a Tumor evaluation at the stage of the initial CCA diagnosis (October 2015, was done with liver vein contrasting (7.5 ml Gadovist). Sum of diameters of target lesions equals $221 \mathrm{~mm}$; b tumor progression after the best clinical practice care (July 2016). Sum of diameters of target lesions equals $278 \mathrm{~mm}$ ( $26 \%$ increase); c tumor growth during Sorafenib treatment (October 2016). Sum of diameters of target lesions equals $314 \mathrm{~mm}$ (16\% increase); d tumor growth after Sorafenib treatment prior to start of Pazopanib (January 2017). Sum of diameters of target lesions equals 392 mm (35.3\% increase). e Tumor progression after Pazopanib treatment (July 2017). Sum of diameters of target lesions equals $471 \mathrm{~mm}$ (35.7\% increase if counting a as a reference or $20 \%$ increase if counting $\mathbf{d}$ as a reference) expression was measured using microarray hybridization. Gene expression data was next used for calculating pathway activation scores and for building rating of target drugs using bioinformatical software Oncobox.

Performed analysis demonstrated that Ras and ERK signaling pathways were highly activated in the patient's pathological tissue (Additional file 1: Table S1). These molecular pathways are implicated in various process linked with tumorigenesis such as cell proliferation, differentiation, survival and apoptosis [28]. Ras pathway activates ERK pathway, but also is tightly connected with stress response, cell motility and cytoskeleton rearrangements [29]. Normally both, ERK and Ras pathways are activated by binding of extracellular growth factors (like EGFR) to their receptor tyrosine kinases (RTKs). TKIs are capable of targeting RTKs, thus inhibiting cell proliferation and survival [30, 31].

Based on the observed molecular phenotype several TKIs were predicted to be effective in the current clinical case (Table 2). Considering both results of the bioinformatical analysis and available literature data on TKI efficiency in CCA, Sorafenib and then Pazopanib were prescribed to our patient. These drugs overlap in blocking FLT1, FLT4, KDR, C-Kit proto-oncogene and platelet derived growth factor receptor beta (PDGFRB). In addition, Pazopanib targets platelet derived growth factor receptor alpha (PDGFRA), while Sorafenib-B-Raf, $\mathrm{C}$-Raf and ret proto-oncogenes, fibroblast growth factor receptor 1 (FGFR1) and FLT3. Both Pazopanib and Sorafenib are approved for treating advanced renal cell carcinoma (RCC); in addition, Sorafenib is approved for treatment of patients with unresectable hepatocellular carcinoma (HCC).

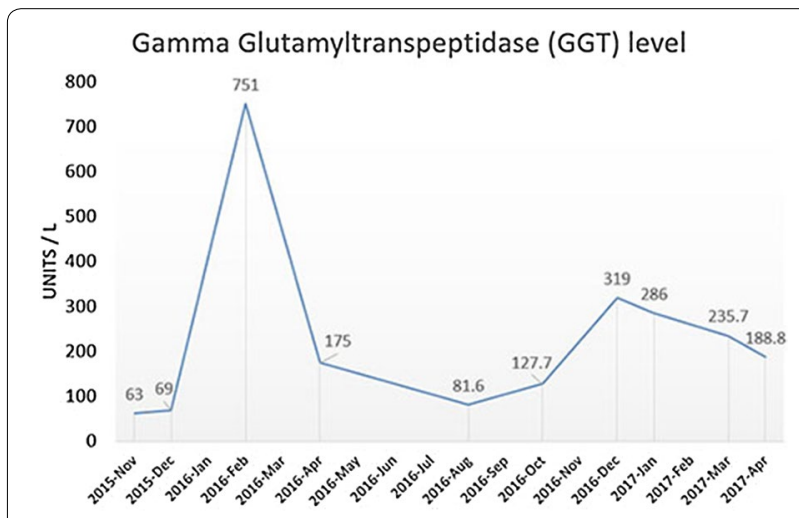

Fig. 3 Serum gamma glutamyltranspeptidase levels during treatment 


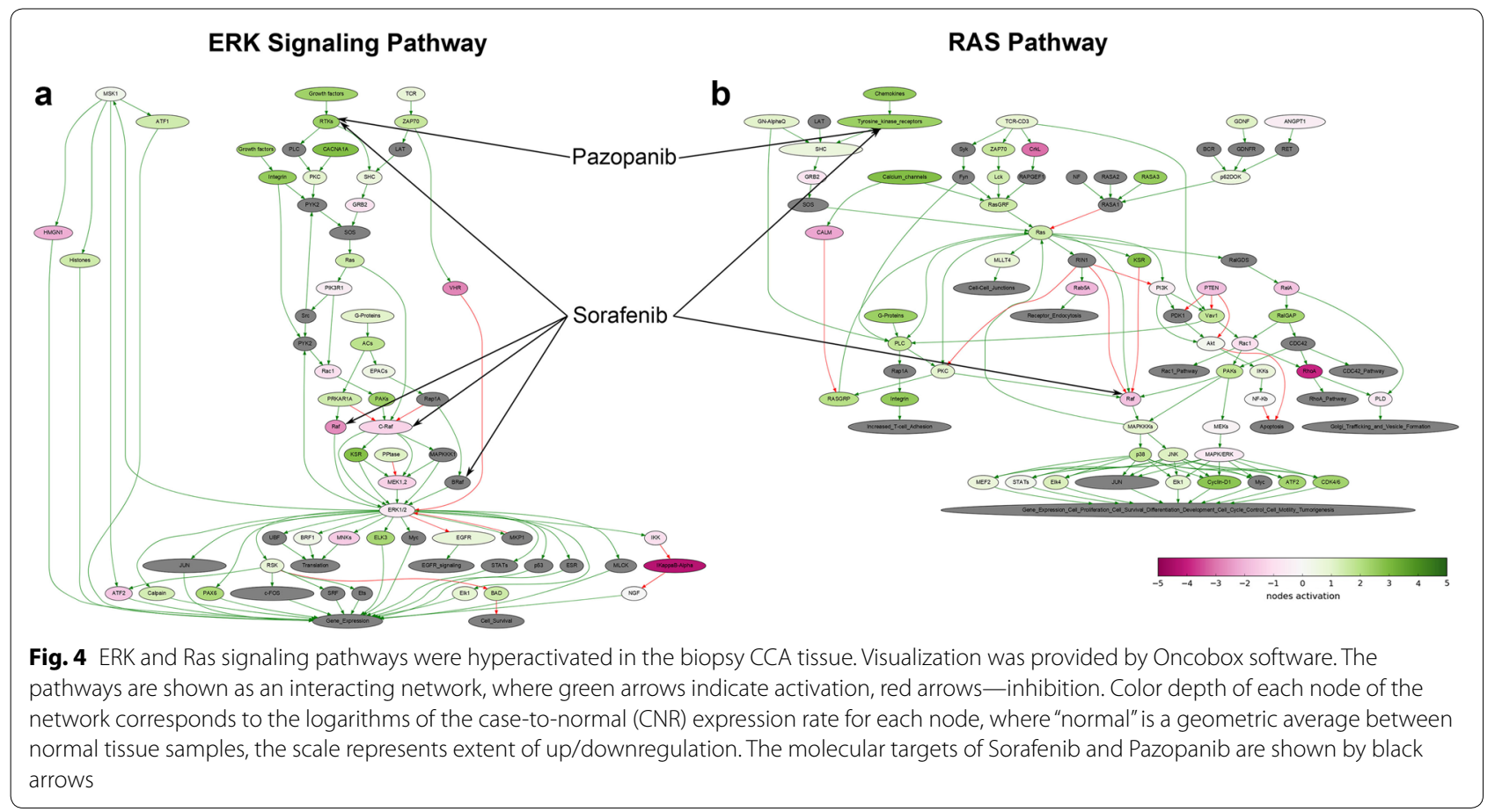

Thus, the performed analysis supported choice of treatment and predicted high chances of TKI efficiency for our patient. Even though the best clinical outcome across this case was progressive disease (according to RECIST), TKIs were beneficial for palliative care of metastatic CCA patient due to improvement of clinical parameters such as quality of life and survival. In addition, start of treatment with TKIs coincided with a trend towards decrease of serum GGT level.

The success of the most clinical trials of TKI in CCA was evaluated based on the time to progression or progression free survival and, therefore, disease progression was considered as an unsuccessful outcome and resulted in termination of treatment. In this case we

Table 2 Rating of target drugs provided by Oncobox test

\begin{tabular}{ll}
\hline Position & Drug \\
\hline 1 & Regorafenib \\
2 & Sorafenib \\
3 & Sunitinib \\
4 & Pazopanib \\
5 & Axitinib \\
6 & Vandetanib \\
7 & Cabozantinib \\
8 & Imatinib \\
9 & Ziv-aflibercept \\
10 & Dasatinib \\
\hline
\end{tabular}

report life quality improvement and prolonged survival with TKI therapy on the background of disease progression. This may point to the modest antitumor activity of TKIs in some patients, as also suggested by El-Khoueiry et al. [16]. Such activity, even if not capable to trigger the response, could potentially be sufficient for slowing down disease progression and, thus, life quality improvement.

\section{Additional file}

Additional file 1: Table S1. Additional table.

\section{Authors' contributions}

$E P, M B, D A, A K, P S$ collected and interpreted patient data. EP and MB were involved in clinical management. MSu performed molecular analyses. MSu, MSe, AA, EA and EP wrote this manuscript. All authors read and approved the final manuscript.

\section{Author details}

${ }^{1}$ I.M. Sechenov First Moscow State Medical University (Sechenov University), Moscow 119991, Russia. ${ }^{2}$ Clinical Center Vitamed, 10, Seslavinskaya St., Moscow 121309, Russia. ${ }^{3}$ Pathology Department, Morozov Children's City Hospital, 4th Dobryninsky Lane 1/9, Moscow 119049, Russia. ${ }^{4}$ State Research CenterBurnasyan Federal Medical Biophysical Center of Federal Medical Biological Agency, Moscow 123098, Russia. ${ }^{5}$ Stanford University School of Medicine, Stanford, CA 94305, USA. ${ }^{6}$ D. Rogachev Federal Research Center of Pediatric Hematology, Oncology and Immunology, Moscow 117198, Russia.

\section{Competing interests}

The authors declare that they have no competing interests. 


\section{Data deposition and access}

Gene expression data derived from the patient's tumor tissue were deposited at the Gene Expression Omnibus (GEO; https://www.ncbi.nlm.nih.gov/geo/) under the Accession Number GSE107233.

\section{Ethics approval and consent to participate}

The patient provided consent for gene expression analysis of his sample and oral consent for the publication of this article. Gene expression profiling was approved by Institutional Review Board (IRB) at Clinical Center Vitamed, Moscow, Russia, according to the Declaration of Helsinki principles.

\section{Publisher's Note}

Springer Nature remains neutral with regard to jurisdictional claims in published maps and institutional affiliations.

Received: 5 April 2018 Accepted: 28 August 2018

Published online: 06 September 2018

\section{References}

1. Bergquist A, von Seth E. Epidemiology of cholangiocarcinoma. Best Pract Res Clin Gastroenterol. 2015;29(2):221-32.

2. Rizvi S, Gores GJ. Pathogenesis, diagnosis, and management of cholangiocarcinoma. Gastroenterology. 2013;145(6):1215-29.

3. Everhart JE, Ruhl CE. Burden of digestive diseases in the United States part III: liver, biliary tract, and pancreas. Gastroenterology. 2009;136(4):1134-44.

4. Yusoff AR, et al. Survival analysis of cholangiocarcinoma: a 10-year experience in Malaysia. World J Gastroenterol. 2012;18(5):458-65.

5. Khan SA, et al. Guidelines for the diagnosis and treatment of cholangiocarcinoma: an update. Gut. 2012;61(12):1657-69.

6. Thongprasert $\mathrm{S}$. The role of chemotherapy in cholangiocarcinoma. Ann Oncol. 2005;16(Suppl 2):93-6.

7. Valle J, et al. Cisplatin plus gemcitabine versus gemcitabine for biliary tract cancer. N Engl J Med. 2010;362(14):1273-81.

8. Feng KC, et al. Cocktail treatment with EGFR-specific and CD133-specific chimeric antigen receptor-modified $T$ cells in a patient with advanced cholangiocarcinoma. J Hematol Oncol. 2017;10(1):4.

9. Castellarin $\mathrm{M}$, et al. Driving cars to the clinic for solid tumors. Gene Ther. 2018;25:165-75.

10. Ahn DH, Bekaii-Saab T. Biliary cancer: intrahepatic cholangiocarcinoma vs. extrahepatic cholangiocarcinoma vs. gallbladder cancers: classification and therapeutic implications.J Gastrointest Oncol. 2017:8(2):293-301.

11. Chakunta HR, et al. Cholangiocarcinoma: treatment with sorafenib extended life expectancy to greater than 4 years. J Gastrointest Oncol 2013;4(4):E30-2

12. Pinter $M$, et al. Sorafenib in unresectable intrahepatic cholangiocellular carcinoma: a case report. Wien Klin Wochenschr. 2011;123(1-2):61-4.

13. LaRocca RV, et al. Effective palliation of advanced cholangiocarcinoma with sorafenib: a two-patient case report. J Gastrointest Cancer. 2007;38(2-4):154-6
14. Luo $X$, et al. Effectiveness and safety of sorafenib in the treatment of unresectable and advanced intrahepatic cholangiocarcinoma: a pilot study. Oncotarget. 2017:8(10):17246-57.

15. Pan TT, et al. A single-center experience of sorafenib monotherapy in patients with advanced intrahepatic cholangiocarcinoma. Oncol Lett. 2017;13(5):2957-64

16. El-Khoueiry AB, et al. SWOG 0514: a phase II study of sorafenib in patients with unresectable or metastatic gallbladder carcinoma and cholangiocarcinoma. Invest New Drugs. 2012;30(4):1646-51.

17. Bengala $C$, et al. Sorafenib in patients with advanced biliary tract carcinoma: a phase II trial. Br J Cancer. 2010;102(1):68-72.

18. Shroff RT, et al. The oral VEGF receptor tyrosine kinase inhibitor pazopanib in combination with the MEK inhibitor trametinib in advanced cholangiocarcinoma. Br J Cancer. 2017;116(11):1402-7.

19. Andersen JB, et al. Genomic and genetic characterization of cholangiocarcinoma identifies therapeutic targets for tyrosine kinase inhibitors. Gastroenterology. 2012;142(4):1021-31.

20. Guo J, et al. Safety of pazopanib and sunitinib in treatment-naive patients with metastatic renal cell carcinoma: asian versus non-Asian subgroup analysis of the COMPARZ trial. J Hematol Oncol. 2018;11(1):69.

21. Yin $X$, et al. Elevation of serum gamma-glutamyltransferase as a predictor of aggressive tumor behaviors and unfavorable prognosis in patients with intrahepatic cholangiocarcinoma: analysis of a large monocenter study. Eur J Gastroenterol Hepatol. 2013;25(12):1408-14.

22. Zhang $C$, et al. Serum liver enzymes serve as prognostic factors in patients with intrahepatic cholangiocarcinoma. Onco Targets Ther. 2017:10:1441-9.

23. Buzdin AA, et al. Oncofinder, a new method for the analysis of intracellular signaling pathway activation using transcriptomic data. Front Genet. 2014;5:55.

24. Artemov A, et al. A method for predicting target drug efficiency in cancer based on the analysis of signaling pathway activation. Oncotarget. 2015;6(30):29347-56.

25. Buzdin A, et al. Activation of intracellular signaling pathways as a new type of biomarkers for selection of target anticancer drugs. J Clin Oncol. 2017:35(15 suppl):e23142.

26. Neuzillet $\mathrm{C}$, et al. Sunitinib as second-line treatment in patients with advanced intrahepatic cholangiocarcinoma (SUN-CK phase II trial): safety, efficacy, and updated translational results. J Clin Oncol. 2015;33(3 suppl):343.

27. Shroff RT, et al. Pazopanib (P) and trametinib (T) in advanced cholangiocarcinoma (CC): a phase Ib study. J Clin Oncol. 2015:33(15 suppl):4072.

28. Wada T, Penninger JM. Mitogen-activated protein kinases in apoptosis regulation. Oncogene. 2004;23(16):2838-49.

29. Simanshu DK, Nissley DV, McCormick F. RAS proteins and their regulators in human disease. Cell. 2017;170(1):17-33.

30. Gollob JA, et al. Role of Raf kinase in cancer: therapeutic potential of targeting the Raf/MEK/ERK signal transduction pathway. Semin Oncol. 2006:33(4):392-406.

31. Sonpavde G, Hutson TE. Pazopanib: a novel multitargeted tyrosine kinase inhibitor. Curr Oncol Rep. 2007:9(2):115-9.

\footnotetext{
Ready to submit your research? Choose BMC and benefit from:

- fast, convenient online submission

- thorough peer review by experienced researchers in your field

- rapid publication on acceptance

- support for research data, including large and complex data types

- gold Open Access which fosters wider collaboration and increased citations

- maximum visibility for your research: over $100 \mathrm{M}$ website views per year
}

At BMC, research is always in progress.

Learn more biomedcentral.com/submissions 